\title{
Transportation, Communication and Sustainability: In Search of a Pathway to Comparative Research
}

\author{
William R. Black (Indiana University) \\ and \\ Peter Nijkamp (Free University, Amsterdam)
}

\begin{abstract}
This paper maps out recent trends in transportation, communication and mobility, against the background of the need for comparative research on a Transatlantic basis. Particular attention is given to the importance of the ICT sector. Questions on comparability and transferability are dealt with, in particular from a sustainability perspective. The paper is concluded with an overview of future research challenges.
\end{abstract}

Pn124bb-RM 


\section{Transportation, Mobility and Communication}

The past decades have witnessed an enormous and unprecedented increase in the volume of intercontinental transport (e.g., between Europe and North-America, and between Asia and NorthAmerica). Wide-body airplanes and mega container ships have shaped the conditions under which our world is becoming smaller all the time. Interestingly enough, a similar development is taking place at a local level, where the action radius is not only increasing, but also the frequency of trips. Geographically, our world is becoming less global and distant, but on the contrary more local and close-by.

Since the early history of mankind, people have tried to expand their geographical action radius. Spatial mobility, either on a daily basis (e.g. commuting) or on a structural basis (e.g. migration), has become a prominent feature of human behavior. With the advent of the Industrial Revolution, new and unprecedented opportunities emerged for extending the spatial range of human activity, thanks to the train, the steamship, the telegraph, the car and the airplane. The 'homo economicus' has also become a 'homo mobilis'.

Nowadays, human behavior is largely synonymous with movement. The old dream of the flying carpet has not yet fully materialized, as the distance friction costs have not vanished, but we are gradually moving toward a space-economy where the death of distance tends to become - to some degree at least - reality. Admittedly, bridging physical distance still incorporates costs (i.e. financial, psychological, time), but over the years these costs tend to decline gradually. Clearly, distance friction continues to play a role in agglomeration phenomena, as geographic proximity (i.e. physical, socio-psychological) generates a great variety of economies of density that compensate for geographic interaction costs. Economies of density seem to run parallel to economies of motion.

More interaction and communication between different parts of our world (at all geographic scales) prompt also an interconnectivity between previously distinct markets. Markets for goods tend to get not only a global uniformity, but they are also becoming spatially more competitive. With the advent of trade liberalisation (thanks to the WTO, the enlargement of the EU, the NAFTA etc.) more physical movement of people and goods is unavoidable.

In our modern network, one cannot imagine an advanced economy without spatial mobility and interaction. And, with an increasing number of people on our earth, more people are becoming more mobile. Since the 1970s, the world population has risen from 4 billion to 6.5 billion, and the US population from approx. 200 million to 300 million. In the same period, the number of vehicles in the US almost doubled, while the total mileage by all cars in the US has almost tripled since the 1970s. These figures illustrate the mobility explosion in our world, but also highlight some of the 
negative externalities (pollution, fatalities, congestion, etc.) of a mobile world. Though there is a phase difference with respect to other countries (e.g., in Europe or in Asia), the pattern of rapid increase in car ownership and use is largely similar all over the world.

Mobility as a key feature of a modern society takes place at all geographic scales. In the past decades, we have not only seen a drastic increase of transport at metropolitan or local levels, but also witnessed a globalization of trade and transport of goods, a phenomenon induced by the fact that mass and scale have more impact on the transport prices of goods than distance. Consequently, the smart organization of goods flows, ranging from local to global scales by means of sophisticated logistics, has become an enormous challenge for transport operators. In this context, the rise of information and communication technology (ICT) has been a breakthrough.

The emergence of the ICT era has indeed prompted a new phenomenon, viz. the transport of information and data on a world-wide scale at almost zero costs. The emerging e-society has had and will continue to have - far-reaching impacts on our modes of production, consumption, knowledge acquisition, and leisure. In recent years, we have witnessed the emergence of interesting scenarios where virtual transportation takes over physical movement. So far, however, we have not observed a substitution between physical movement and virtual transport (i.e., telecommunication). In fact, both phenomena are still on a rising edge and seem to exhibit - at least at a macro scalecomplementarity.

Transportation and communication have developed over the centuries in a dependent manner, in which the former was very much a precursor of the latter. The transmission of information was, for all practical purposes, a transportation process as well as a communication process. Letters and packets sent during the centuries prior to 1844 moved at the same speed as the mode of transport, which therefore dictated the speed of communication, whether the carriers were post riders, stage coaches, or sailing ships. Although pigeons had been used since the time of the Roman Empire for short messages, Samuel Morse's invention of the telegraph in 1844 was probably the first significant break in this communication-transport dependence. Of course, the spread of the telegraph was also intimately involved with the spread of the railroad, the transport mode for which schedules and time were far more important than other early modes. The Atlantic cable of the 1860s and Bell's telephone invention of the 1880s further widened the developing chasm between transport and communication.

This dependence continues to the present day, when the rapid development of ICT has not only meant an enormous rise in productivity caused by our information and communication economy but has also facilitated the better logistic handling of both passenger and goods transportation. Nevertheless, even though the technological drivers of our mobile world share common features in 
many countries, there may be different patterns of mobility among various countries as a result of varying adoption mechanisms of new technologies, different lifestyles and residential living patterns, different land use patterns and different transportation policies (see Stead 2003). Therefore, it is important to investigate the commonalities and contrasts in the transport field in different countries more thoroughly. In particular, at the transatlantic edge we witness rather drastic differences in both behavior and policy, which warrants an ambitious effort to draw lessons from comparative study. Comparative transportation analysis would need systematically collected databases, not only on actual transport movements but also on transport policies and the negative impacts of transportation (such as congestion).

The above mentioned trends prompt a scientific investigation along two complementary lines. First, the rapid increase in transatlantic transport calls for applied, modelling and policy research on actual flows, impediments to mobility, risks of large-scale transport volumes (e.g., security), modern logistics and ICT, as well as policy arrangements on international transport (e.g., open skies or open seas agreements). In the second place, almost all countries face similar problems associated with the mobility revolution (such as congestion, environmental decay, traffic fatalities, just-in-time logistics). Such issues demand a thorough analysis and an exchange of experiences from different regions in our world could be extremely useful in finding solutions and remedies for mobility impediments, not only in the field of technological progress but also in the area of behavioural adjustments. From a policy perspective, attempts to establish new forms of policy coordination, to ensure a liberalisation of the transport markets, to develop new forms of effective traffic management, or to design an environmentally-benign lay-out of new urban areas would lead to intriguing questions on comparability and transferability of policy lessons between e.g. Europe and the United States.

It is no surprise that the dynamics in transportation and communication has prompted a search for new research tools in the form of operational methods and models. The present volume offers an interesting collection of innovative approaches. Our introductory chapter aims to provide a contextual exploration of new analytical perspectives for understanding the drivers of the above mentioned dynamics. We will address in particular the opportunities for the comparative analysis of mobility flows and interests in Europe and North America, while taking into account differences in technological development paths, in regulatory trajectories and in behavioural responses at both the supply and demand side.

The remainder of this contribution is therefore organized as follows. In Section 2, we will address the strong relationship between transport and communication, as well as the negative externalities of transport in a mobile society; and the analytical potential of scenarios in an uncertain 
environment will also be highlighted. Next, Section 3 will address the complex interdependency between ICT and sustainable transport, while Section 4 will focus on the sustainability of ICT. Then Section 5 will then pay attention to similarities and differences in transportation, as seen from a transatlantic perspective. That section also discusses the associated possibilities for comparative research on the transatlantic edge. Finally, Section 6 will make some suggestions regarding a future research agenda.

\section{Transport and Communication}

The railroads of the early 20th century were the primary mode of transport for moving mail between major cities. In the United States this led to the creation of railway post offices (RPOs): rail cars where mail was processed and bagged for distribution at various points along the railroad's route. These RPOs actually had their own cancellation equipment that carried the name of the railroad on the cancelled postage. Although these mobile post offices continued for several decades, they ceased to be of real importance probably by the middle of the century.

The use of wireless radio communication became common in the early years of the century. It was useful for ensuring travel safety and the scheduling of arrivals for different transport modes, but not for much else.

During this same period, various inventors and entrepreneurs were experimenting with the development of automobiles. Innovations in this area were evident on both sides of the Atlantic, and it was ultimately Ford's assembly line that enabled the internal combustion engine to win out over the electric and steam automobiles of the day. Although the automobile brought about many changes, its development is notable here as a transport mode that was initially completely independent of communication.

World War I saw the further development of motorized vehicles. Airplanes also developed during this era and, in the beginning, these too functioned without any communication. In the immediate post-war period we saw the development of airmail, a faster way of moving communication than the railroad at that time, but the latter was still used for the movement of express mail at night.

Communication technology was used as early as 1929 in aviation. In this case radio beams were used to assist pilots with navigation. During the 1930s, radio communication began to appear in aircraft and this was significant in the general field of aviation safety and as a device to assist with navigation. It was in the 1940s, after World War II, that radar became common in the commercial aviation sector for air traffic control and as a way of monitoring aircraft location, landings, take-offs, and so forth — once again, a significant safety improvement. 
Major changes in communication capabilities of the other transport modes were slow. The motor carriers began using citizen band radios, but these were not major developments. By midcentury, a new set of problems was emerging. These were problems brought on by the rapid growth of the automobile and the rise of commercial aviation. From this time until the end of the $20^{\text {th }}$ century, these problems for the most part simply increased.

Such problems were, however, the proverbial tip of the iceberg in terms of what we refer to today as problems of sustainable transport. At that time, these new problems began to manifest themselves as problems with local air quality due to automotive exhaust emissions, general problems attributable to, and defined by, congestion, and, in its wake, problems of equity. Although the first of these needs no explanation, it was in attempts to solve the congestion problems that the equity problem arose. These equity problems had to do primarily with the household relocation effects created by attempts in the U.S. and elsewhere to build more highways as a solution to the congestion problem.

The availability of petroleum also emerged as a problem of transportation sustainability. Petroleum is the major fuel source for all motorized transport. The distribution of this resource is not uniform, and it is anticipated that reserves of this fuel may be depleted by the mid- $21^{\text {st }}$ century. In addition, the developed world is very much dependent on the developing world for this resource but, in many cases, the former lacks the control of the resource that it would like and this also has an impact on its transport sustainability.

Fatalities and injuries had been increasing since the beginning of the $20^{\text {th }}$ century, though very few viewed this as something that could be resolved. During that century, in the U.S. motor vehicle accidents resulted in more than three million fatalities. By the 1960s, concern was being expressed about the safety of motor vehicles and this concern led first to the use of seat belts and much later air bags (see also WHO 2004).

In the U.S., the equity problem was resolved partially by the Relocation Assistance Act which remedied many of the early equity problems. But local air quality problems have still not been resolved and congestion continues to be a major transport problem globally. In addition, there has been greater recognition of the association between global warming and carbon dioxide emissions.

A mobile society undoubtedly offers a wide variety of positive outcomes, such as better access to facilities, more opportunities for social interaction, and many trade gains. Some of these outcomes may be regarded as positive externalities in an economic sense. Recently, however, there has been an overwhelming interest in the shadow side or disadvantages of large-scale transport, in particular in the negative externalities (Gudmundsson 2003; Nijkamp and Vleugel 1995; Steg 2003).

These negative externalities have prompted extensive research, e.g. on the assessment of the 
social costs of transport (incurred by congestion, fatalities, pollution, landscape destruction etc.). The ongoing debate on the feasibility of road pricing exemplifies the complexity of the issues involved. In recent years, many studies have also addressed the technological uncertainty in future transportation systems (such as those using electric cars, zero-emission cars, hydrogen vehicles, etc.). To map out the future (or at least the intrinsic uncertainties of the future), many researchers and policymakers have resorted to scenario experiments (see Van Geenhuizen and Nijkamp 2003).

Scenarios could play an interesting role in complex decision issues, as scenario analysis is in agreement with the desire to unfold a panorama of possible futures, which are probably generated in the light of past causalities, and offer an integrated future picture of part of a complex reality. They can be seen as coherent mappings of likely, feasible or desirable future states of (a part of) society, including the trajectory between the present and the future. Scenarios are different from policy strategies, in that they cannot be chosen by policymakers, in contrast to policy strategies. Strategies are the responses to exogenously given developments. There are two types of scenarios, viz. those which look forward and those which are of a backcasting nature. The use of scenarios has several advantages for decision making under uncertainty and risky decisions, because scenarios:

- are a rational way to judge future uncertainty in a cohesive way;

- depict uncertainty from the viewpoint of driving forces;

- offer an intellectual framework for a sensitivity analysis in terms of robustness of strategies;

- are strategic learning tools for decision makers;

- may act as communication tools in an uncertain multi-actor situation;

- stimulate creative thinking on an open future.

Scenario analysis - including in the transportation policy sector - has gained much popularity. In Nijkamp et al. (1998) several approaches to scenario building in the transportation sector are presented. In their study they used a distinction of future images of transportation into expected and preferred developments, using survey techniques among a wide range of transportation experts. The estimated transport implications of these images (in terms of person kms or tonne kms) were next translated into emissions of air pollution, through which tests on sustainability consequences could be carried out.

Various classes of scenario building experiments may be distinguished. From the perspective of the involvement and views of the designer, one may distinguish inter alia: intuitive scenarios, idealistic scenarios, qualitative scenarios, quantitative scenarios and participatory scenarios. From the perspective of application fields of scenarios, one may distinguish, inter alia, projective and prospective scenarios, autonomous (background) scenarios, reference (trend) scenarios, surprise-free and challenge scenarios, contrast (borderline) scenarios, and conflict scenarios. In all cases, 
scenarios are analytical and visionary mental constructs. They are not necessarily valid, but provide a frame for thinking in a rational way about the future. They are meant to prompt proper action in terms of policy strategies, but are not intended to play a role as valid predictions.

In recent years, various extensive scenario studies on the feasibility of future sustainable transport systems have been undertaken. Examples can be found in, amongst others, Banister et al. (2000), Nijkamp et al. (1998), and Rodenburg et al. (2001). These studies not only presented a systematic constellation of transport scenarios at different geographic scale levels, but also an exploration of policy strategies aimed at achieving more sustainable development. Policy instruments and measures to be used are, for instance, regulation, subsidies, and fair and efficient pricing.

\section{ICT and Sustainable Transport}

ICT entered the transport sustainability battle in the mid-20th century. Traffic signals on expressway ramps that controlled the entrance of vehicles onto major highways and freeways were controlled by television signals that informed central traffic control operators of the density of vehicles on these roads. In this way, the signals were controlled and this prevented vehicle density from increasing to congestion levels, at least some of the time.

Since then, the involvement of ICT in the area of transport sustainability has continued. The entire area of air traffic control is essentially an ICT field. It controls the flights of all commercial aircraft and most general aviation traffic as well. Its fundamental importance is well-illustrated by the complete paralysis of this network when the system goes down for one reason or another. This is one example of the manner in which transport has become virtually dependent on communication technology. This is a complete reverse of the situation of 150 years ago when virtually all communication relied on transportation.

Congestion control in motor vehicle transport has also continued to develop over the years. The television cameras of mid- $20^{\text {th }}$ century are now suspended above thousands of intersections. Instead of an individual monitoring all this, the system is set to change signals when it perceives a traffic jam building up. Humans are not involved after the equipment is installed, unless things go wrong. Communication with the drivers takes place through the use of the traffic signal. There are also demand-activated signals where the presence of one or more vehicles at a signalized intersection is "perceived" by sensors in the highway surface. The signal responds depending on where the volume is the greatest and this facilitates traffic flow and reduces congestion.

Within urban areas, it has been common to use cameras to record vehicles proceeding against a stop traffic signal for some time. This technology is also being used to keep track of vehicles 
moving within certain areas where congestion fees apply. This is the case in London where a fee was initiated in February 2003 for motor vehicles moving within a defined central area of that city during certain times of the day. Currently, there are discussions about ultimately increasing this zone to include all areas within the M25 orbital highway that surrounds London, but this will most likely require the use of a different technology: the use of transponders and cell-tower based technology.

Satellite and cell-tower technology are also being considered as a way of identifying the road use taxes due from highway, road and street users in the United States. Hitherto, these taxes were based on gallons of gasoline sold, but the improvement in the fuel efficiency of current vehicles and the increasing use of alternate fuels and hybrid fuel vehicles will decrease the revenues received. A global positioning system (GPS) and a geographic information system (GIS) along with transponders would enable users to be taxed on the basis of miles over which their vehicle used different types of roadways. Prototypes of this system are currently being tested in the U.S..

A similar GPS/GIS system is used in the more expensive motor vehicles being sold today. This system has the capability of communicating directly with the driver of these vehicles in the event that an air bag deploys. It also has the capability of dispatching local emergency vehicles to the location of the vehicle. In addition, the system can be used to assist with navigation and in this way decrease fuel use and emissions attributable to way-finding.

Nowadays, in several European cities, sensors record the entry and exit of vehicles into multilevel garages, and information about the availability of parking spaces is sent to electronic displays along highways. This prevents drivers from wandering through the street system in search of a parking space, adding to congestion, fuel use and pollution.

Still further examples of ICT involvement with transportation are on the horizon with the use of front, rear, and side radar on vehicles. These innovations would virtually prevent vehicles from running off the road or into other vehicles. The sensors would inform the driver of the approaching difficulties and, in the process, decrease the risk of accidents, injuries, and fatalities, and this would make transport more sustainable.

ICT has thus various implications for the transport sector. It may help to encourage greater efficiency in transport, but in the long run it may also stimulate more mobility, as intensified interaction and communication tends to create more physical transport. But is may do so at lower environmental costs.

As the above examples demonstrate, ICT is making transport far more sustainable than it would otherwise be. It also is making the current and future transport system far more dependent on ICT. A word of caution is in order here. The above sketch of the potential of ICT was mainly supply- 
oriented and offered an optimistic picture. But institutional, political, behavioural and logistic bottlenecks may preclude a full achievement of all the potential benefits of ICT. The limited success of these potentials in countries like Germany, France or Italy is illustrative. This raises the question: Just how sustainable is ICT? In the realm of transport we recognize resource depletion, congestion, injuries and fatalities, and emissions as problems arising from transport not being sustainable. Are there similar or analogous situations in the realm of ICT?

\section{The Sustainability of ICT and Transport}

Certain nations of the world today do not have sufficient petroleum to power the fleets of vehicles that operate on their transport systems. They find that they are essentially at the mercy of other nations who may control the availability and price of this fuel. The imposition of oil embargoes of the 1970s was one of the primary reasons why the U.S. government began to look at alternate fuels at that time. Today, certain nations find that if they base control of their public systems (possibly transport systems) on ICT software available only from major vendors, then they are virtually controlled by the vendor concerned. The lack of standardization and the unavailability of computer source code in much software makes it impossible for most users to modify it and, as a result, whenever the vendor upgrades or alters its software, or switches technologies, the user is obliged to make the same changes. This is hardly a sustainable situation and it is very significant in the developing nations of the world.

The major attempt to counter this problem has been the development of free and open-source software (FOSS). FOSS is software for which the source code has been made public and may be altered by the user. Aside from enabling countries to be independent of vendors, it also allows these same countries to develop expertise in the area as programmers to see the manner in which certain systems are put together. Programs may be modified for specialized uses and then shared with other users. Most of this software has been developed in such a way that it will run on virtually any machine.

We usually do not think of the world of ICT as having accidents resulting in fatalities and injuries to humans using such a system. In the case of motor vehicles, these accidents are generally attributable to three fundamental causes: human error, environmental factors and design defects. The question is whether these causal factors could also have an impact on ICT and consequently on its sustainability. If experience to date is representative, then these facors could also play a role in the realm of ICT. Programming errors (i.e. human errors) will most likely be found in the software of any system and situations arise that are never anticipated. Some of these (for example, digitizing errors) have already occurred and been corrected, but others will undoubtedly occur in the future as 
new data input is added to information systems.

Environmental errors may be particularly daunting since reliance on GPS satellite systems is subject to blackouts caused by solar activity. This is quite disturbing today when one watches television relayed by satellites, but it could actually be hazardous if that GPS is monitoring or controlling the movement of vehicles.

Design defects are less obvious, but just as much of a potential problem. We can not, at this point, anticipate what exactly these might be, for if we had such prescience they would not occur.

ICT will definitely influence spatial behaviour and hence impact on the transport of people and goods. The latter aspect deserves indeed due attention, as the emerging e-society and sophisticated logistics will most likely shape new patterns of spatial interaction.

Congestion in the transport sector must also have a counterpart in the communication sector, but this is rarely discussed. Concern here is not with the additional geosynchronous satellites that may have to be placed in orbit, but rather with the potential confusion of the multitude of signals to and from these satellites. What are the potential problems that could occur? How secure would such systems be? These are just a couple of the questions that one might pose if we begin to rely too heavily on ICT as a way of moving toward transport sustainability.

One final area in which ICT is playing a role in improving the sustainability of transport and travel is the substitution of communication for travel or movement. There are several areas in which this is viewed as having some potential. The first of these is in the straight substitution of a communications link-up for workers travel to their place of work. This is referred to as telecommuting in the U.S. and teleworking in Europe. 'Teleworking' is actually the better term since such employees are actually working at a distance.

There is no doubt that teleworking actually is possible, and that numerous employees in the U.S. and Europe do practice this type of substitution. However, it is very unlikely that they are teleworking five days a week. Because of this, it is reasonable to ask: is this limited use of teleworking actually enough to decrease accidents, congestion, emissions, and fuel use? Probably not, but this does not mean teleworking is insignificant. The major problem is that there are still too few jobs to which it can be applied.

Another form of substitution is the replacement of a certain amount of travel by e-commerce. Recent data suggests that e-commerce in the U.S. and EU is approximately $1.5 \%$ of total retail sales in each area. Under the premise that every little bit helps, we do not want to cast this completely aside, but we also should not expect this to be a source of significant reduction in motor vehicle traffic. How much of the e-commerce taking place is merely a substitution of the Internet for sales that were previously done by telephone or mail-order or catalog sales? If this is the case, is this type 
of substitution having any impact at all on the transport sector?

Certainly e-commerce in the business-to-business transactions is much more significant; it represents about $15 \%$ of such transactions. But, once again, how much of this business was previously done over telephone lines and, therefore, does it really represent a reduction in transportation? Probably not. There is already a significant increase in small-scale deliveries of parcels etc. as a result of internet use for shopping.

To summarize, this section has looked at the historical linkages between transportation and communication and noted the manner in which the virtual dependence of the latter on the former for most of recorded history has changed in the last 150 years in such a way that communication has become independent of transport. This independence continued through the first half of the $20^{\text {th }}$ century, but transport then began to depend more and more on communication and information technology (ICT) in response to problems in the area of transportation sustainability.

At present, the role of ICT in moving toward a more sustainable transport system is becoming of growing importance and one must query whether ICT is sustainable, or, as in the case of transport, if we increase our dependence on this sector, will it also become non-sustainable. ICT may have significant impacts on spatial behaviour and hence on urban interaction patterns and in the long run on urban structure, by allowing more dispersion. Preliminary analysis would suggest that is the potential outcome.

Also important from many perspectives is the substitution of communication in areas of the journey to work and in the purchasing of retail goods; these are teleworking, in the former case, and e-commerce in the latter. In the area of teleworking, we find the number of individuals involved is often too small in most cases to have a major impact on any of the factors that make transport nonsustainable: congestion, accidents, fuel use, and emissions. In the area of e-commerce we conclude that the substitution is merely of one form of communication for another, and the transport portion of the commerce is little affected by this change.

A final remark concerns aviation. The airline sector is strongly driven by the ICT sector, and will continue to be structurally influenced by new developments in ICT. This will generate more long-distance mobility, while ensuring higher levels of sustainability. Lessons from the airline sector (e.g., safety requirements) will increasingly be adopted by other transport sectors.

\section{Similarities and Differences in Transportation and Communication: Toward a Transatlantic Comparative Perspective}

All countries in our world seem to be moving toward higher mobility levels. Less developed countries may have lower mileage figures, but sooner or later they tend to catch up, as is witnessed 
in European mobility data. Seen from this perspective, both North America and Europe might be expected to exhibit similar mobility phenomena. At first glance, this may seem to be true, but a closer investigation also reveals some interesting differences in terms of the structure of mobility patterns and related policy.

In the first place, it ought to be recognized that North America has a clear private car orientation that is instigated by individual market beliefs, whereas Europe has more a mixed (private car and public transport) orientation which makes the operation of the transport market different from that in North America. Second, a prominent feature is also that - as part of the North American life style public transport does not play an essential role, and is mainly oriented towards specific target groups, whereas in Europe public transport has a great deal of public support and forms an indispensable part of the total transport system. And, finally, the transport market in Europe tends to be seen to a considerable extent as a public sector responsibility, while in North America liberal market perspectives tend to prevail.

In recent years, comparative study has become an enormous methodological challenge in many disciplines, as it may reveal important lessons from available information. The presence of extensive databases has undoubtedly contributed to the current popularity of comparative analysis in a quantitative sense, in particular, meta-analysis.

Comparative analysis may address relevant features of a complex phenomenon under consideration. For example, it may refer to the inputs of a process (e.g., expenditures for infrastructure) in order to assess their efficiency. But it may also address the performance of a system by investigating output indicators (e.g. the occurrence of congestion). And finally, comparative research may address the impacts of policies (e.g. the effect of road pricing schemes on congestion). In all such studies, the main aim is to identify causal or explanatory patterns in the functioning of a common set of complex features which exhibit considerable variation in space and time. Comparative studies may originate from various sources of interest, such as testing a causal relationship; identifying whether a proposition in a given study is also applicable in other studies; exploring whether a critical condition in a given case result also holds somewhere else; or observing whether there are commonalities in causal structures and in empirical results in different case studies. It goes without saying that the structure and the development of transportation on both sides of the Atlantic is particularly suitable for quantitative comparative research.

The main traditional method for a synthesis of research findings in economic research has been the literature review. As a method of research it is well developed. According to Van den Bergh et al. (1997), surveys have often tended to report findings in a tabular or narrative fashion with verbal comments and a discussion of the strengths and weaknesses of each study. Although useful, such 
studies are qualitative in character and neglect a full account of many quantitative aspects of the individual studies. Neither can they fully account for the difficulties underlying the application of synthesis, such as the incompatibility of pieces of knowledge.

In 1976, Glass introduced the, then, new study of meta-analysis. Originally, this study approach, which finds its origin in experimental psychology, aimed at examining a well-defined collection of experiments in an integral way by using statistical methods. This study process has evolved towards a broader field of application. Meta-analysis has also found its way into economics, where an increasing number of quantitative studies are available.

In the literature many definitions of meta-analysis have been presented thus far. However, none of these is entirely satisfactory, and, in view of the necessity for a solid methodological underpinning, we offer a more precise definition. We consider meta-analysis as a scientific investigation of a well-defined collection of previously presented individual (case)studies concerning a certain phenomenon, in which (mainly) quantitative research methods are applied which are able to test and assess qualitative as well as quantitative knowledge based on a well-defined collection of available study material with the aim to (i) gain new insight into the chosen phenomenon under study, and (ii) achieve a quantitative research experiment.

The use of meta-analysis in economics has resulted in a rising tide of scientific contributions. But, clearly, it has also increasingly been recognized that economics is at best a quasi-experimental science, so that external study circumstances are hard to control. For example, the year of data collection, the geographical location, the sample size, or the functional forms of models in various empirical studies are usually not equal or uniform, and may have significant impacts on the study outcomes. In many cases, empirical studies were not intended for comparative analysis, so that statistical inference from a sample of previous studies is highly problematic. It is thus of critical importance to design an operational methodology that accounts to the maximum extent possible for differences in study design and execution. In general, meta-analysis is a proper methodological vehicle for those research questions that are investigated in comparable study designs and formats. Only then, can meta-analytical procedures generate additional information from an existing body of knowledge, by deploying proper methods for the quantitative research synthesis of findings from a range of prior studies.

In recent years, we have seen an avalanche of comparative studies and meta-analyses in the field of transportation. These contributions have highlighted the analytical vigor and have applied lessons from research syntheses in this field.

\section{Prospect for a Research Agenda}


There is certainly a need for thorough comparative analysis in the transportation sector. Despite different research traditions, different policy constellations, different lifestyles and mobility patterns and different socioeconomic conditions, it is of the utmost relevance to identify commonalities and contrasts in research findings and policy impacts. The following issues may serve as illustrations of a possible research agenda for comparative study:

- general transportation issues

Examples are access conditions to (public and private) transport, differences in environmental stress, contrasting views on mobility and social rights, or different shares of passenger and freight transport.

- problem identification in regard to the human dimension

In this setting, one may want to analyze and design megatrends of mobility (e.g. individualization, ageing, economic growth), the organization of our space-economy (urbanization, land use, etc.), or people's innate drive to benefit from the 'flying carpet' idea (see Section 1).

- strategic policy issues

Here one may think of policy scenario design for sustainable transport; the identification of constraints, goals and opportunities for sustainable mobility; or the exploration of different pathways and transitional roads to sustainable transport systems (e.g. hardware, information provision, attitude changes, or shifts in policy practices).

- social well-being and human behavior

The following issues may illustrate this approach: the assessment of values and perceptions of the 'homo mobilis'; the social dilemma emerging from efficiency, equity, and quality in transport access; or the modeling of the social epidemiology of noise, stress or safety.

- policy handles and policy research

Important concerns in this context are: the transparent modelling of individual and collective interests in mobility behaviour, the assessment of sustainable transport impacts and perception by different socioeconomic target groups, or the systemic innovation of our land use-transportation systems (including industrial site development, satellite cities, etc.).

It goes without saying that a proper analysis and sophisticated modeling exercise of this complex force field means an enormous challenge for the transportation research community. There is clearly a need for a transition from speculative arguments to solid theory, from subjective reflections to testable models, from aggregate macro-oriented analysis to behavioural, micro-based experimental models, and from a mono-disciplinary approach to a creative exploration of the opportunities for sustainable mobility at the edges of different disciplines. And, most of all, there is a clear need for systematic fact-finding, leading to consistent and harmonized empirical databases so 
as to pave the road for international comparative research. This would be a sine qua non for a mature comparative study program on European and North-American mobility and transportation patterns, and elsewhere in the world.

\section{References}

Banister, D., D. Stead, P. Akerman, A. Dreborg, P. Steen, P. Nijkamp and R. Schleicher-Tappeser, European Transport and Sustainable Development, SPON, London, 2000

Bergh, J.C.J.M. van den, K.J. Button, P. Nijkamp and G. Pepping, Meta-analysis in Environmental Economics, Kluwer Academic, Dordrecht, 1997

Geenhuizen, M. van and P. Nijkamp, Coping with Uncertainty: An Expedition into the Field of New Transport Technology, Transportation Planning and Technology, vol. 26, no. 6, 2003, pp. 449-467

Glass, G.V., Primary, Secondary and Meta-analysis of Research, Educational Research, vol. 5, 1976, pp. 3-8

Gudmundsson, H., Making Concepts Matter: Sustainable Mobility and Indicator Systems in Transport Policy, International Social Science Journal, no. 176, 2003, pp. 199-218

Hensher, D.A. and K. Button (eds.), Handbook of Transport and the Environment, Elsevier, Amsterdam, 2003

Nijkamp, P. and J. Vleugel, In Search of Sustainable Transport Systems, European Transport and Communications Networks (D. Banister, R. Capello and P. Nijkamp, eds.), John Wiley, Chichester, 1995, pp. 287-299

Nijkamp, P., S. Rienstra and J. Vleugel, Transportation Planning and the Future, John Wiley, New York, 1998

Rodenburg, C.A., B. Ubbels and P. Nijkamp, Open Windows of Europe, European Economic and Political Issues III (F. Columbus, ed.), Nova Science Publishers, New York, 2001, pp. 117-136

Stead, D., Transport and Land-Use Planning: Really Joined Up? International Social Science Journal, no. 176, 2003, pp. 333-348

Steg, L., Can Public Transport Compete with the Private Car?, IIATS Research, vol. 27, no. 2, 2003, pp. 27-36

WHO, Report on Traffic Accidents; see http:/www.who.int/world-health-day/2004/infomaterials/world_report/en/ chapter1.pdf 